\title{
Quality Control of the Foot Revascularization Using Indocyanine Green Fluorescence Imaging
}

\section{Settembre, Nicla}

2017-07

Settembre , N, Kauhanen , P , Albäck , A , Spillerova , K \& Venermo , M 2017 , ' Quality Control of the Foot Revascularization Using Indocyanine Green Fluorescence Imaging ' , World Journal of Surgery , vol. 41 , no. 7 , pp. 1919-1926 . https://doi.org/10.1007/s00268-017-3950-6

http://hdl.handle.net/10138/236999

https://doi.org/10.1007/s00268-017-3950-6

unspecified

publishedVersion

Downloaded from Helda, University of Helsinki institutional repository.

This is an electronic reprint of the original article.

This reprint may differ from the original in pagination and typographic detail.

Please cite the original version. 


\title{
Quality Control of the Foot Revascularization Using Indocyanine Green Fluorescence Imaging
}

\author{
Nicla Settembre $^{1} \cdot$ Petteri Kauhanen $^{1} \cdot$ Anders Albäck $^{1} \cdot$ Kristyna Spillerova $^{1}$. \\ Maarit Venermo ${ }^{1}$
}

Published online: 6 March 2017

(C) Société Internationale de Chirurgie 2017

\begin{abstract}
Objectives Critical limb ischemia (CLI) is a clinical diagnosis, confirmed by objective tests, usually ankle-brachial index (ABI), toe pressure (TP) and TcPO2. Furthermore, the anatomical lesions in patients affected by CLI were visualized by ultrasound, angiography, CTA, or MRA. Indocyanine green fluorescence imaging (ICG-FI) is a diagnostic modality for assessing foot perfusion. We aimed to study the usefulness of ICG-FI in the quality control of revascularization.

Materials and methods One hundred and four CLI limbs in 101 patients were studied with ICG-FI using SPY Elite before and after open or endovascular revascularization. ABI and TP were also measured. After ICG-FI, assessment of circulation was done using time-intensity curve derived from the two regions of interest the one being in the plantar side of the foot and the other in the dorsal side of the foot. Three parameters were derived from the curves: maximum intensity (the absolute value of the maximum intensity); intensity rate (the value from the time-intensity curve describing the increase in maximum intensity/s) and SPY10 (the intensity achieved during the first $10 \mathrm{~s}$ after the foot starts to gain intensity).

Results Sixty-two limbs presented category 3 of Rutherford classification, 12 limbs category 4, and 30 limbs category 5. Ninety-five technically successful procedures were achieved, $63(66.3 \%)$ endovascular and $32(33.7 \%)$ surgical revascularizations. In $9(9.5 \%)$ patients, an in-line flow from the aorta to the foot was not achieved due to a failure to recanalize the occlusion $(n=7)$ or due to distal embolization $(n=2)$. ABI was not reliable in 58 patients $(57.4 \%)$ mostly due to pseudohypertension and TPs in $49(48.5 \%)$ patients mostly due to previous minor amputations. ICG-FI was successful in all patients. The mean intensity values before and after the procedure in patients who underwent successful revascularization were $81 \pm 47$ units and $120 \pm 5$ units of intensity $(p<.001)$ and intensity rates $4.2 \pm 4$ and $8.0 \pm 6.2$ units/s $(p=.001$ ), respectively. In the PTA patients in whom the revascularization was unsuccessful, no changes were seen in the hemodynamic parameters. In $6(8.8 \%)$ patients who underwent technically successful revascularization, the SPY values were worse after the revascularization than at the baseline.

Conclusions ICG-FI with SPY Elite provides reliable information on the increase in perfusion after revascularization, in addition to implicating possible failure if there is no improvement in the ICG-FI variables. Unlike ABI and $\mathrm{TP}$, it can be performed in all patients. It gives valuable information to complement traditional assessment methods.
\end{abstract}

Nicla Settembre

nicla.settembre@yahoo.com

1 Department of Vascular Surgery, Helsinki University Hospital, University of Helsinki, P.O. Box 340, 00029 HUS, Helsinki, Finland

\section{Introduction}

Critical limb ischemia (CLI) is a significantly morbid disease that increases the risk of amputation and mortality. The prevalence of CLI is increasing as the population ages and the incidence of diabetes grows. Revascularization is 
recommended to obtain wound healing and limb salvage [1].

CLI is a clinical diagnosis, but it should be supported by objective tests. Multiple methods are utilized to determine foot perfusion and the extent of arterial lesions, including the ankle-brachial index (ABI), toe pressure (TP) and $\mathrm{TcPO} 2$, as well as Doppler measurement, duplex ultrasound scanning, and other more invasive procedures such as a CT scan and angiography [1]. Furthermore, the assessment of the increase in the perfusion after revascularization is important. After bypass surgery, this is usually evident and a "red hot foot" tells about a successful procedure. However, after endovascular revascularization the situation is often not so evident. After technically successful PTA, the clinical picture of the wound and foot may be unchanged and toe pressures may not show huge increase or in some cases they are impossible to measure [2].

Fluorescent angiography using indocyanine green (ICG) has been demonstrated in reconstructive surgery to identify territories of adequate and inadequate perfusion [3, 4] in order to decrease complications and increase the early detection of ischemic tissues on the flaps [5]. Near-infrared fluorescence imaging is a noninvasive and non-ionizing diagnostic modality for assessing the perfusion of tissues. Although some publications exist on the use of fluorescence imaging in the assessment of ischemic feet, the technique is uncommon in vascular surgery. Few studies are available on patients with peripheral arterial disease [6-9]. Terasaki et al. [6] introduced the use of two parameters, PDE10 and T1/2 derived from the time-intensity curve, to be used in the assessment of foot perfusion. PDE10 is the intensity of the fluorescence $10 \mathrm{~s}$ from the origin of the curve, and T1/s is the time in seconds that is needed to achieve half of the maximum intensity.

In the current study, we utilized the fluorescence imaging technique in the assessment of foot perfusion before and after revascularization in patients with CLI. The aim was to study the usefulness of ICG imaging in the immediate quality control of revascularization and the sensitivity of the technique to distinguish early failures in the revascularization. Furthermore, we analyzed the differences in the increase in the perfusion between surgical bypass and endovascular revascularization.

\section{Materials and methods}

Altogether 750 infrainguinal endovascular procedures and 200 surgical bypasses due to CLI are performed in our institution annually. During September 2015 and March 2016, 101 patients and 104 limbs treated with infrainguinal endovascular or surgical revascularization due to CLI were studied with an indocyanine green fluorescence imaging using SPY device (SP 3055 Novadaq $^{\circledR}$ ) before and after revascularization (Fig. 1). The system is equipped with an $806 \mathrm{~nm}$ light-emitting diode (LED) as an excitation light source and a charge-coupled device (CCD) camera covered with a lens that filters out light with a wavelength of $830 \mathrm{~nm}$.

Data on the patients' risk factors and the status of the ischemic foot as well as the details of the endovascular or surgical revascularization were collected prospectively. One-third $(29 \%, n=30 / 104)$ of the patients had previous revascularization in history (31\% of the surgical and $28 \%$ of the endovascular patients). Data on atherosclerotic lesions in lower-limb arteries were collected from either magnetic resonance angiography or digital subtraction angiography.

\section{Assessment of the hemodynamic changes achieved with revascularization}

$\mathrm{ABI}$ and $\mathrm{TP}$ were measured in all patients preoperatively and after revascularization. It was not possible to measure $\mathrm{ABI}$ mostly due to the uncompressible arteries at the ankle level both before and after the revascularization in 58 patients $(57.4 \%)$ and toe pressures mostly due to previous minor amputations in $49(48.5 \%)$ patients. ICG-FI was successful in all patients, and it was performed before and after revascularization.

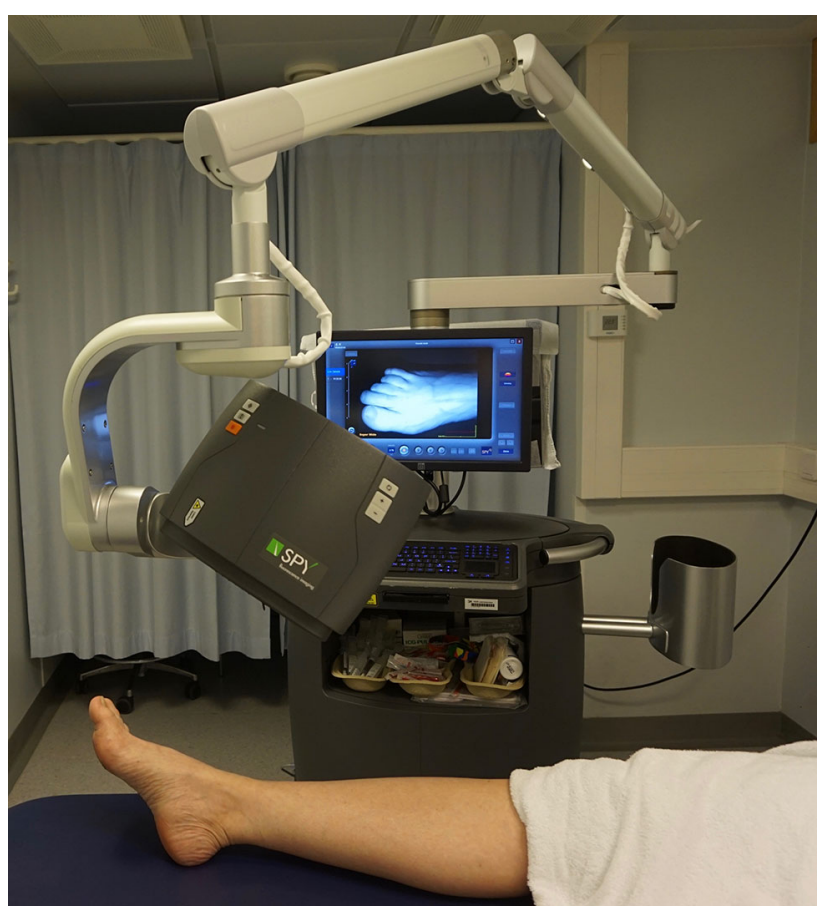

Fig. 1 Setup for the ICG-FI. In a darken room, a SPY camera is positioned above the foot of the patient and ICG-FI is recorded 
The measurements were taken in a room with no daylight and a temperature of $20-25{ }^{\circ} \mathrm{C}$. After a 15 -min rest, the patients lay in the supine position. The ICG-FI camera was positioned $20 \pm 3 \mathrm{~cm}$ above the plantar region of the foot. ICG $(0.1 \mathrm{mg} / \mathrm{kg})$ was injected intravenously to the brachial vein, and a 4-min continuous recording was immediately initiated. Twenty minutes after the first recording was finished, the examination was repeated on the dorsum of the foot. The 20-min break between the two examinations was held in order to let the ICG from the first study to disappear from the body before the new examination.

The SPY Elite device provided a time-intensity curve of the selected region of interest (ROI) showing the fluorescence intensity plotted against time in seconds [10] (Fig. 2). Three parameters were derived from the timeintensity curve: (1) maximum intensity, which is the absolute value of the maximum intensity in the time-intensity curve; (2) intensity rate, which is the value from the time-intensity curve describing the increase in maximum intensity per seconds; and (3) Spy10, which is the intensity achieved during the first $10 \mathrm{~s}$ after the foot starts to gain intensity. Two ROIs were used in all cases - the first in the dorsum of the foot and the second in the plantar region. The mean value of these two measurements was used for the analysis. The variables extracted from the time-intensity curve of the ICG-FI were compared with ABI and TP.
During the ICG-FI recording, an automatic baseline setting allowing adjustment for possible confounding factors, such as possible daylight or residual ICG in the second measurement, was used.

The study was approved by the Ethics Committee of our institution. Informed consent was not asked as the ICG-FI is in routine use for patients with CLI in our department.

\section{Statistical analysis}

SPSS 22.0 was used in statistical analysis. The continuous variables are expressed as mean $\pm \mathrm{SD}$ and range. Dichotomous variables are expressed as percentages. Comparison of the continuous variables before and after revascularization was made using repeated samples $t$ test. The results were analyzed separately for the endovascular procedures (PTA group) and surgical revascularization (bypass group).

\section{Results}

The mean age of the patients was $76.2( \pm 11.2)$ years, and $53.8 \%$ have diabetes. The basic demographics of the study patients and anatomical details of the revascularizations are presented in Table 1. Sixty-two limbs presented category 3 of Rutherford classification [11], 12 limbs category 4, and
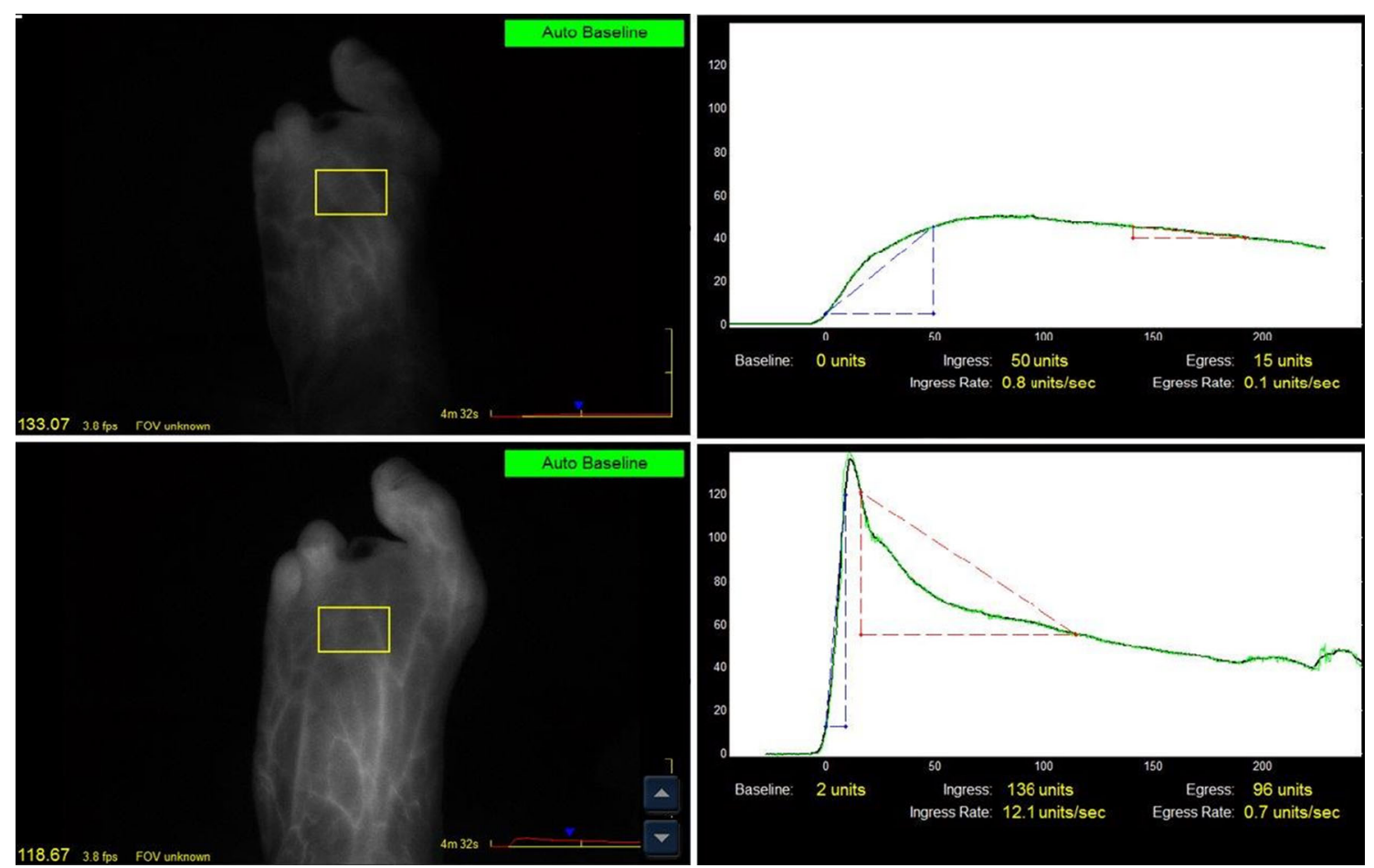

Fig. 2 ICG-FI image and time-intensity curve drawn of the region of interest before (above) and after (below) the revascularization showing intensity (ingress) and intensity (ingress) rate values (time-intensity curve, SPY-image, regions we measured) 
Table 1 Basic demography of the patients and anatomical details of the revascularization

\begin{tabular}{llll}
\hline & Bypass & PTA & Total \\
\hline Number of patients/limbs & $33 / 33$ & $68 / 71$ & $101 / 104$ \\
Age (years \pm SD) & $74 \pm 11$ & $76 \pm 11$ & $76 \pm 11.2$ \\
Gender $n(\%)$ & $12 \mathrm{~F} \mathrm{(36.4)}$ & $40 \mathrm{~F}(58.8)$ & $52 \mathrm{~F}(51.5)$ \\
& $21 \mathrm{M}(63.6)$ & $28 \mathrm{M}(41.2)$ & $49 \mathrm{M}(48.5)$ \\
Diabetes $n(\%)$ & $18(54.5)$ & $36(50.7)$ & $56(55.4)$ \\
Hypertension $n(\%)$ & $30(90.9)$ & $57(80.3)$ & $90(89.1)$ \\
Cardiac risk factors $n(\%)$ & $10(30.3)$ & $22(31.0)$ & $32(31.7)$ \\
Dyslipidemia $n(\%)$ & $31(93.9)$ & $56(78.9)$ & $90(89.1)$ \\
Stroke $n(\%)$ & $6(18.2)$ & $10(14.1)$ & $16(15.8)$ \\
Active smoking $n(\%)$ & $7(21.2)$ & $13(18.3)$ & $20(19.2)$ \\
Previous smoking $n(\%)$ & $13(39.4)$ & $13(18.3)$ & $26(25.0)$ \\
SFA revasc. $n(\%)$ & $0(0)$ & $10(14.7)$ & $10(9.6)$ \\
Popliteal artery revasc. $n(\%)$ & $16(48.5)$ & $15(22.1)$ & $31(29.8)$ \\
Crural revasc. $n(\%)$ & $17(51.5)$ & $46(67.6)$ & $63(60.6)$ \\
\hline
\end{tabular}

SFA superficial femoral artery, $F$ female, $M$ male

30 limbs category 5. Of the 104 procedures, $10(9.6 \%)$ were performed to SFA, $31(29.8 \%)$ to popliteal artery, and $63(60.6 \%)$ to crural arteries. In $9(9.5 \%)$ legs, an open line from the aorta to the foot was not achieved due to a failure to recanalize the occlusion $(n=7)$ or due to distal embolization $(n=2)$. These 9 procedures were analyzed separately. There were no immediate technical failures of bypass surgery. Of the 95 technically successful procedures, 63 (66.3\%) were endovascular and 32 (33.7\%) surgical revascularizations.

The mean ABI increased from the baseline $0.41( \pm 0.14$, range $0.17-1.04)$ to a $0.85( \pm 0.2$, range $0.22-1.38)$ $(p<.001)$ after the revascularization, and toe pressures increased from $29( \pm 12$, range $12-125)$ to $49( \pm 20$, range 15-103) $(p=.07)$.

ICG injections were well tolerated by all patients, with no adverse events related to this procedure. The mean intensity values before and after the procedure in patients who underwent successful revascularization were $81 \pm 47$ units (range 15-242) and $120 \pm 5$ units (range 27-245); mean intensity rate values were $4.2 \pm 4.9$ (range 0.1-25.1) units/s before the revascularization and $8.0 \pm 6.2$ (range 0.2-31.3) units/s after the revascularization $(p=.001)$. SPY10 intensity was $26 \pm 26$ units (range $1-139)$ and $59 \pm 42$ units (range $2-203)(p<.001)$.

In the 9 patients in whom the endovascular revascularization was unsuccessful, no changes were seen in the hemodynamic parameters. The changes in hemodynamic parameters after failed and successful endovascular procedures and surgical repair are presented in Table 2 .

In $6(6.3 \%)$ patients, who underwent technically successful revascularization, the SPY values were worse after the revascularization than at the baseline. Three of them underwent a distal puncture of the crural vessel and the stenosis was recanalized from a retrograde direction (Fig. 3). Later, duplex ultrasound showed thrombosis of the artery distal to the puncture, explaining the SPY results. In $3(3.2 \%)$ bypass patients, the SPY variables were worse after the revascularization than at the baseline. In two of them, the bypass was occluded, and one had a significant inflammation of the foot when the preoperative SPY was performed, causing a falsely good time-intensity curve.

\section{Discussion}

Revascularization in patients affected by CLI improves leg salvage and survival. However, most of the patients need continuous surveillance of the revascularization and the foot to achieve wound healing and to prevent amputation. Furthermore, it is important to ensure the result of the revascularization by measuring the arterial perfusion before and after the revascularization. The conventional methods for assessing the perfusion of the foot in patients with critical limb ischemia are the ankle-brachial index (ABI) and toe pressure [1]. The use of these methods with TcPO2 can be helpful to make a quantitative analysis of foot perfusion. Although these methods are validated by TASCII [1], their use is, to a degree, limited, and they cannot always be applied [12, 13].

An ICG-FI is a noninvasive modality for assessing the perfusion of the tissues. It is easy to use and safe [14, 15]. Several studies have demonstrated the benefits of fluorescence imaging in medical applications such as retinal angiography, liver clearance testing, and cardiac output monitoring, but also in neurosurgery as well as cardiac, 
Table 2 Analysis of 104 limbs

\begin{tabular}{|c|c|c|c|c|c|}
\hline Technique/number of patients & Type of examination & Mean & Number of patients & SD & SE mean \\
\hline \multicolumn{6}{|l|}{ Paired samples statistics } \\
\hline BYPASS & SPY ingress before & 77.79 & 33 & 50.0 & 8.70 \\
\hline \multirow[t]{9}{*}{33 limbs } & SPY ingress after & 134.17 & 33 & 51.96 & 9.04 \\
\hline & SPY ingress rate before & 4.86 & 33 & 6.12 & 1.06 \\
\hline & SPY ingress rate after & 10.15 & 33 & 5.28 & 0.92 \\
\hline & SPY10 before & 26.58 & 33 & 33.93 & 5.90 \\
\hline & SPY10 after & 69.74 & 33 & 34.39 & 5.98 \\
\hline & ABI before & 0.41 & 11 & 0.14 & 0.04 \\
\hline & ABI after & 0.63 & 11 & 0.29 & 0.08 \\
\hline & TP before & 29.08 & 12 & 12.07 & 3.48 \\
\hline & TP after & 30.33 & 12 & 12.84 & 3.70 \\
\hline PTA & Ingress before & 83.33 & 62 & 46.35 & 5.88 \\
\hline \multirow[t]{9}{*}{62 limbs } & Ingress after & 113.07 & 62 & 55.94 & 7.10 \\
\hline & Ingress rate before & 3.91 & 62 & 4.15 & 0.52 \\
\hline & Ingress rate after & 6.80 & 62 & 6.30 & 0.80 \\
\hline & SPY 10 before & 25.96 & 62 & 20.37 & 2.58 \\
\hline & SPY 10 after & 53.96 & 62 & 44.45 & 5.64 \\
\hline & ABI before & 0.65 & 26 & 0.21 & 0.42 \\
\hline & $\mathrm{ABI}$ after & 0.85 & 26 & 0.20 & 0.40 \\
\hline & TP before & 41.15 & 33 & 22.39 & 3.89 \\
\hline & TP After & 49.54 & 33 & 20.77 & 3.61 \\
\hline Technical failure PTA & Ingress before & 109.07 & 9 & 52.79 & 17.59 \\
\hline \multirow[t]{9}{*}{9 limbs } & Ingress after & 83.9 & 9 & 57.13 & 19.04 \\
\hline & Ingress rate before & 5.35 & 9 & 4.96 & 1.65 \\
\hline & Ingress rate after & 2.83 & 9 & 4.23 & 1.41 \\
\hline & SPY 10 before & 24.0 & 9 & 20.17 & 6.72 \\
\hline & SPY 10 after & 18.7 & 9 & 22.68 & 7.56 \\
\hline & ABI before & 0.58 & 6 & 0.21 & 0.08 \\
\hline & ABI after & 0.72 & 6 & 0.21 & 0.08 \\
\hline & TP before & 40.29 & 7 & 38.65 & 14.60 \\
\hline & TP after & 46.42 & 7 & 25.71 & 9.71 \\
\hline
\end{tabular}

Three groups were separately studied

reconstructive, and general surgery [16]. However, only few studies exist on the ICG in vascular surgery $[6-8,17]$, and even less is known about the use of SPY in patients with critical limb ischemia. In the current study, we evaluated the use of ICG-FI in the assessment of an ischemic foot as well as in the quality control of revascularization in 109 patients. We demonstrated that SPY is useful, especially when studying the change in circulation after revascularization. Furthermore, it has some advantages over the conventional methods and provides more reliable quantitative information on the perfusion of the foot.

The concept of ICG-FI is relatively old but has not been utilized in vascular surgery until recently [8]. We have previously used another ICG-FI device [6]. SPY Elite is an evolution of the other infrared camera; however, it maintains the same principles. Indeed, the main changes are found in the software for analyzing the results, making them easier to achieve and therefore even faster to analyze. The analysis of perfusion in the ROI is more accurate with the new device, owing to the better resolution of the camera. With the SPY-Q software, a single frame of each recording can be measured in units of absolute perfusion applied to the ROI. The unit of absolute perfusion is based on a standardized scale pixel gray gradient from 0 to 255 . The automatic baseline setting was used for all patients in order to compensate for any differences in intensity and for residual IC in the circulatory system that might bias the quantitative assessment and visualization. In addition, the 

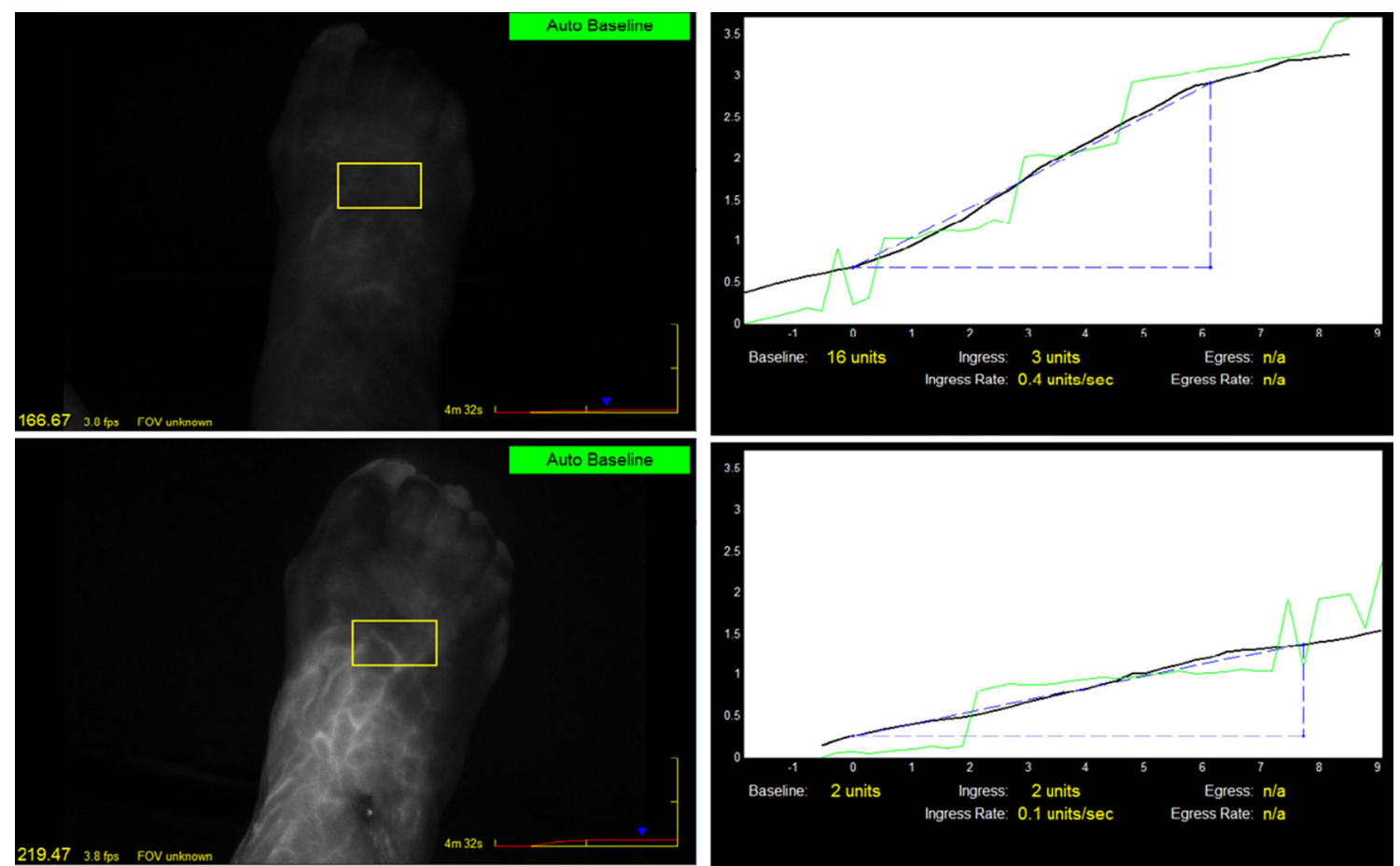

Fig. 3 Time-intensity curve for the first $10 \mathrm{~s}$ before (top) and after (below) the revascularization in a patient who underwent recanalization of anterior tibial artery using pedal access. ICG-FI shows poorer intensity in forefoot distal to the puncture site

equipment includes an arm for holding the camera, which provides different positions of the camera related to the foot.

In order to assess the vascularization of our patients, for this study, one of the applications of the SPY-Q program was used that allows the comparison of the curves of perfusion before and after the procedure. We analyzed the values of intensity and intensity rate in each ROI before and after the revascularization. The analysis was based on measurements taken over the entire exam and during a span of $10 \mathrm{~s}$ to provide a comparative display of the perfusion of the foot. Terasaki [6], in a study on patients with peripheral arterial disease, described a perfusion time of $10 \mathrm{~s}$ in normal healthy patients; this time, which does not depend on the device, was adopted as the reference value in our study to graphically quantify an index of good perfusion in the selected ROIs. The intensity value reports the total change in blush from the start to maximum values. The "intensity rate" describes the average rate at which the blush changes during the intensity. ICG with a SPY device has been reported for the intraoperative assessment of perfusion in plastic and reconstructive surgery. Clinical studies have demonstrated the ability of SPY analysis to preoperatively evaluate any zones of the flap suffering from ischemia to reduce the risk of postoperative complications and re-interventions.
The potential utility of this new method remained unknown to vascular surgeons for a long time. Terasaki et al. [6] published quantitative information about the perfusion of the area of interest, first describing a quantitative evaluation using ICG fluorescence in patients with lower-limb arterial diseases. Unno et al. [7] used ICG to assess the patency of preoperative bypass in 6 patients, demonstrating the absence of the passage of green into the occluded bypass. In a small study, Braun et al. [7] used SPY Elite before and after revascularization in 13 patients. They reported significant increase in the intensity after the revascularization in all patients and concluded that this method provides rapid qualitative visual and quantitative information about regional foot perfusion.

By comparing the preoperative and postoperative intensity curves in the same ROI, it is possible to evaluate the perfusion of the foot. In our series of patients in whom PTA or bypass was successful, SPY analysis revealed that the intensity as well as the intensity and intensity rate increased, while in patients whose PTA was unsuccessful, the intensity and/or IR remained unchanged or with nonsignificant modifications. The statistical analysis revealed that, for some patients, the intensity and an intensity rate decreased after the procedure. These results suggest that the perfusion of the foot became worse after surgery. Indeed, analyzing the individual cases, worse distal perfusion was related to distal embolization in two cases and a 
thrombosis of the distal pedal artery in one patient who underwent PTA retrograde pedal access, although the final angiographic control showed a patent artery before an introducer sheath retry. The utility of SPY, in these cases, has been to allow the assessment foot perfusion early, within $6 \mathrm{~h}$ after the procedure, permitting the early remedy of postoperative complications with the ability to re-operate on these patients faster.

The values of SPY were compared with ABI and toe pressure before and after the intervention. ABI and toe pressure measurements have some clear disadvantages when compared to ICG-FI. ABI is falsely elevated in many cases, especially in diabetic patients. In the current study, reliable $\mathrm{ABI}$ was available before and after the procedure in only $48(47.5 \%)$ patients. Toe pressures are more reliable but cannot be measured in one-third of patients. Also, in many cases the values are available before the operation but not after the operation as some minor amputations have been done. In this series, only $52(51.5 \%)$ patients had both pre- and postoperative toe pressures. Furthermore, the repeatability of toe pressure measurements is not excellent [2]. With ICG-FI, the perfusion can be assessed from a larger region, and in addition to numerical values, ICG-FI provides visual information, which is very valuable. ICGFI is not reliable in feet with inflammation as the intensity tends to be falsely elevated. Furthermore, if there is necrotic tissue in the foot, SPY does not yield reliable information. ICG-FI can also been used during the operation itself. The SPY device can be covered with a sterile window for that purpose.

In this study, the increase in arterial perfusion as measured by ICG-FI was significantly higher after surgical bypass as opposed to PTA. The same phenomenon can be seen in ABI measurements. The preoperative baseline values were worse in surgical patients, but after the procedure, the level of perfusion seemed to be on the same level.

The ICG-FI image also has several limitations. For example, we are not able to measure the circulation straightly from wound area usually due to necrotic tissue on the surface. Usually, necrotic tissue does not show any fluorescence leading to false-positive finding, on the other hand infection causing inflammation causes hyperemia and false-negative finding regarding ischemia. Furthermore, ICG seems to vary a lot between individuals. This means that although the repeatability of the study is good and comparison of the studies of an individual patient reflects well the changes of perfusion, we have not been able to define any normal values or thresholds for example to critical ischemia. Costs of the ICG-FI equipment vary between 50,000 and 150,000 euro depending on the device and the software. The cost of ICG is about 5-10 euro per patient. Although the cost of the device is high, the net cost is lower if we are able to prevent major adverse limb events with earlier diagnostics on the revascularization failure and restenosis after revascularization.

There are many limitations in our study. Firstly, the number of patients so far is limited, especially in the bypass group. Furthermore, we do not currently have surveillance data, which means that the clinical meaning of the SPY values remains unknown. In the future, we aim to follow these patients and compare the ICG-FI data against their wound healing and ability to predict failure of bypass.

In our series, the results of SPY analysis are in agreement with the real perfusion of the foot. In our experience, this noninvasive analysis can be useful in terms of assessing the perfusion of the foot after distal revascularization by means of PTA or bypass.

We think that ICG-FI may be a useful exploration in patients who undergo distal revascularization due to CLI and tissue lesion, before and after the revascularization and thereafter during the follow-up until the wound is healed. Significant worsening of the ICG-FI is a signal for restenosis and the need of further imagining.

Patients in whom the post-procedure SPY analysis reveals a worse intensity value need further investigations as Doppler ultrasound or new angiography to check for distal embolization or early thrombosis of the revascularized arteries.

\section{Conclusions}

ICG-FI with SPY Elite provides reliable information on increase in perfusion after revascularization, in addition to implicating possible failure in revascularization if there is no improvement in the ICG-FI variables. ICG-FI may help to distinguish possible problem in revascularization earlier, and thus may prevent major adverse limb events. It gives valuable information complement to traditional assessment methods ABI and TP, which are many times not applicable. According to our study, the increase in perfusion is higher when surgical bypass is performed as opposed to endovascular revascularization. Further studies are needed to evaluate the benefit of this new method of follow-up and would healing prediction.

\section{References}

1. Norgren L, Hiatt WR, Dormandy JA, Nehler MR, Harris KA, Fowkes FG, TASC II Working Group (2007) Inter-society consensus for the management of peripheral arterial disease (TASC II). J Vasc Surg 45(Suppl S):S5-S67

2. Widmer LW, Vikatmaa $P$, Aho $P$, Lepäntalo $M$, Venermo $M$ (2012) Reliability and repeatability of toe pressures measured 
with laser Doppler and portable and stationary photoplethysmography devices. Ann Vasc Surg 26(3):404-410

3. Patel KM, Bhanot P, Franklin B, Albino F, Nahabedian MY (2013) Use of intraoperative indocyanin-green angiography to minimize wound healing complications in abdominal wall reconstruction. J Plast Surg Hand Surg 47:476-480

4. Liu DZ, Mathes DW, Zenn MR, Neligan PC (2011) The application of indocyanine green fluorescence angiography in plastic surgery. J Reconstr Microsurg 27:355-364

5. Newman MI, Samson MC, Tamburrino JF, Schwarz KA, Brunworth L (2011) An investigation of the application of laser assisted indocyanine green fluorescent dye angiography in pedicle transverse rectus abdominus myocutaneous breast reconstruction. Can J Plast Surg 19:1-5

6. Terasaki H, Inoue Y, Sugano N, Jibiki M, Kudo T, Lepäntalo M, Venermo M (2013) A quantitative method for evaluating local perfusion using indocyanine green fluorescence imaging. Ann Vasc Surg 27:1154-1161

7. Unno N, Nishiyama M, Suzuki M, Yamamoto N, Inuzuka K, Sagara D et al (2008) Quantitative lymph imaging for assessment of lymph function using indocyanine green fluorescence lymphography. Eur J Vasc Endovasc Surg 36:230-236

8. Braun JD, Trinidad-Hernandez M, Perry D, Armstrong DG, Mills JL Sr (2013) Early quantitative evaluation of indocyanine green angiography in patients with critical limb ischemia. J Vasc Surg 57:1213-1218

9. Venermo M, Settembre N, Albäck A, Vikatmaa P, Aho PS, Lepäntalo M, Inoue Y, Terasaki H (2016) Pilot assessment of the repeatability of indocyanine green fluorescence imaging and correlation with traditional foot perfusion assessments. Eur J Vasc Endovasc Surg 52:527-533
10. SPY-Q Analysis Toolkit Operator's Manual Document: 012-50101-000 Edition: USA Revision: B, 2012 Printed in Canada, pp 51-52

11. Rutherford RB, Baker JD, Ernst C, Johnston KW, Porter JM, Ahn $S$ et al (1997) Recommended standards for reports dealing with lower extremity ischemia: revised version. J Vasc Surg 26(3):517-538

12. Wyman RA, Keevil JG, Busse KL, Aeschlimann SE, Korcarz CE, Stein JH (2006) Is the ankle-brachial index a useful screening test for subclinical atherosclerosis in asymptomatic, middle-aged adults? WMJ 105:50-54

13. De Graaff JC, Ubbink DT, Legemate DA, de Haan RJ, Jacobs MJ (2001) Interobserver and intraobserver reproducibility of peripheral blood and oxygen pressure measurements in the assessment of lower extremity arterial disease. J Vasc Surg 33:1033-1040

14. Cheng Z, Wu Y, Xiong Z, Gambhir SS, Chen X (2005) Nearinfrared fluorescent RGD peptides for optical imaging of integrin alphavbeta3 expression in living mice. Bioconjug Chem 16:1433-1441

15. Frangioni JV (2003) In vivo near-infrared fluorescence imaging. Curr Opin Chem Biol 7:626-634

16. Alander JT, Kaartinen I, Laakso A, Pätilä T, Spillmann T, Tuchin VV, Venermo M, Välisuo P (2012) A review of indocyanine green fluorescent imaging in surgery. Int $\mathrm{J}$ Biomed Imaging 2012:940585

17. Kang Y, Lee J, Kwon K, Choi C (2010) Dynamic fluorescence imaging of indocyanine green for reliable and sensitive diagnosis of peripheral vascular insufficiency. Microvasc Res 80:552-555 